\title{
Suplementación alimenticia de glutamina sobre el desempeño productivo en pollos de engorde
}

\section{Nutritional supplementation of glutamine on the productive per- formance in broiler chickens}

Jimmy Quisirumbay-Gaibor ${ }^{1}{ }^{2 *}$, Raquel Torres ${ }^{1}$, Jhon Yupanqui ${ }^{1}$, Diego Martínez Patiño-Patroni ${ }^{1}$, Carlos Vílchez Perales ${ }^{1}$

Autor para correspondencia: jrquisirumbay@uce.edu.ec

${ }^{1}$ Escuela de Posgrado, Universidad Nacional Agraria La Molina. Av. La Molina s/n La Molina. Lima-Perú.

${ }^{2}$ Facultad de Medicina Veterinaria y Zootecnia, Universidad Central del Ecuador, Ciudadela Universitaria, Jerónimo Leiton s/n y Gatto Sobral. Quito-Ecuador.

\begin{abstract}
Resumen
El objetivo de esta investigación fue evaluar el tamaño del efecto de la suplementación de glutamina sobre el rendimiento productivo de pollos de engorde, la consistencia de su efecto y la influencia de otros factores mediante el uso de meta-análisis. Se encontró que la suplementación de glutamina favorece el aprovechamiento de nutrientes reflejado en menores valores de conversión alimenticia (calculada con ganancia de peso) 1,484 versus 1,518 , y un tamaño medio de efecto de $-0,031$ (diferencia de medias) $(\mathrm{P}=0,00005)$. Los pollos control requieren consumir $31 \mathrm{~g}$ adicionales de alimento para producir $1 \mathrm{~kg}$ de peso ganado en comparación con el grupo que sí recibió el aminoácido en estudio. Cuando la variable analizada fue conversión alimenticia (calculada con peso vivo) (1,468 vs. 1,478), se determinó que los pollos sin glutamina requieren de $15 \mathrm{~g}$ adicionales de alimento para producir $1 \mathrm{~kg}$ de peso vivo (diferencia de medias $=-0,015 ; \mathrm{P}=0,00001)$. No se encontró un efecto consistente entre los estudios realizados, presentándose valores elevados en el test de inconsistencia, 100\% y 81,6\% para las dos formas de conversión de alimento. Las meta-regresiones señalan que factores relacionados al diseño del experimento o ciertos niveles de nutrientes tienen impacto sobre la variable en estudio. Se concluye que la suplementación de glutamina favorece el rendimiento productivo en pollos de engorde. Sin embargo, se debe considerar el diseño del estudio y la formulación de la dieta.
\end{abstract}

Palabras clave: nutrición, alimentación, aves, aminoácidos, conversión alimenticia.

\begin{abstract}
The objective of this research was to evaluate the effect size of glutamine supplementation on the productive performance of broiler chickens, the consistency of their effect and the influence of other factors through the use of meta-analysis. It was found that glutamine supplementation favors the use of nutrients reflected in lower values of feed conversion (calculated with weight gain) 1,484 versus 1,518, and an average effect size of $-0,031$ (difference of means) $(\mathrm{P}=0,00005)$. Control chickens require an additional $31 \mathrm{~g}$ of feed to produce $1 \mathrm{~kg}$ of weight gain compared to the group that did receive the amino acid under study. When the variable analyzed was feed conversion (calculated with live weight) $(1,468$ vs 1,478$)$, it
\end{abstract}


was determined that chickens without glutamine require an additional $15 \mathrm{~g}$ of feed to produce $1 \mathrm{~kg}$ of live weight (difference of means $=-0,015 ; \mathrm{P}=0.00001$ ). No consistent effect was found between the studies carried out, with high values in the inconsistency test, $100 \%$ and $81.6 \%$ for the two forms of feed conversion. The meta-regressions indicate that factors related to the design of the experiment or certain nutrient levels have an impact on the variable under study. It is concluded that glutamine supplementation favors the productive performance in broiler chickens. However, the design of the study and the formulation of the diet should be considered.

Keywords: nutrition, feeding, birds, amino acids, feed conversion.

\section{Introducción}

La glutamina (Gln) es un constituyente importante de proteínas y es un precursor para la síntesis de aminoácidos, nucléotidos, ácidos nucleicos, amino-azúcares y varias otras importantes moléculas biológicas (Roth, 2008). Adicionalmente, la glutamina actúa como un sustrato energético para las células de rápida proliferación como son los enterocitos y linfocitos activos en el epitelio intestinal (Calder \& Yaqoob, 1999; Pohlenz et al., 2012). Como inmuno-nutriente, la glutamina promueve la integridad y maduración de la microbiota intestinal asociada con el sistema inmune, mejorando la síntesis de mucina que mantiene la estructura de la mucosa intestinal y refuerza la barrera epitelial contra el ataque bacteriano (Soares et al., 2014; Yi et al., 2005). La glutamina trabaja como un transportador de nitrógeno, ayuda a defender al organismo del exceso de amoníaco (Labow et al., 2001), está disponible en tejidos, músculos y proteínas, provee energía para el sistema inmune y ayuda al cuerpo a soportar condiciones de estrés (Hu et al., 2016; Newsholme, 2001). Tradicionalmente ha sido considerado como un aminoácido no esencial, sin embargo, ahora aparece como un nutriente condicionalmente esencial durante estados de enfermedad o lesiones serias y puede ser un candidato potencial en el mejoramiento de la respuesta al estrés ya que mejora la expresión de las proteínas de estrés térmico (Phanvijhitsiri et al., 2006; Wischmeyer, 2002). La glutamina es el aminoácido más abundante en el plasma bajo condiciones de salud, sin embargo, las concentraciones caen precipitadamente después de lesiones, cirugía, infecciones u otros estados de estrés (Askanazi et al., 1980; Zhong et al., 2012). Una gran cantidad de investigaciones en modelos animales bajo estados de infección y daño severo han demostrado que la suple- mentación de Gln mejora la sobrevivencia, el estado inmune, disminuye bacteremias, incrementa la función de la barrera intestinal e inhibe la atrofia de la mucosa intestinal (Luquetti et al., 2016; Wang et al., 2014; Wu et al., 2011). En pollos de engorde se ha reportado que la suplementación dietaria de Gln mejora el rendimiento productivo y las características de carcasa bajo situaciones de estrés calórico (Dai et al., 2009; Olubodun et al., 2015; Sifa et al., 2018). La administración in-ovo al día 7 de incubación mejora la ganancia de peso y la conversión alimenticia medida a los 42 días de edad (Salmanzadeh et al., 2016). La Gln ha mostrado reducir la carga de S. typhimurium en contenido cecal al ser comparados frente a un grupo control (Menconi et al., 2013). Varios trabajos reportan que la suplementación a través de la dieta de Gln favorece el desarrollo de las vellosidades intestinales y la consecuente mejora del desempeño productivo (Abdulkarimi et al., 2019; Nascimento et al., 2014; Shakeri et al., 2015; Soltan, 2009). Sin embargo, varios han sido los niveles de inclusión dietaria y las circunstancias experimentales bajo las cuales se han desarrollado estos estudios. El objetivo de este manuscrito fue evaluar el tamaño del efecto de la suplementación de Gln sobre el rendimiento productivo de pollos de engorde, la consistencia de su efecto y la influencia de otros factores mediante el uso de la herramienta estadística de meta-análisis.

\section{Materiales y métodos}

\subsection{Fuente de información (datos)}

Se realizó una búsqueda electrónica de artículos científicos en revistas indexadas con revisión doble ciego en las siguientes bases electrónicas: CAB Direct, 
Elsevier Biobase-CABS, Google Scholar, MEDLINE, PubMed, Science Direct (Journal), Scopus, Academic Search Complete, CAB Abstract, Directory of Open Access Journals. Se utilizó una combinación de palabras clave: glutamina, Gln, dieta, alimento, nutrición, pollos, rendimiento productivo, conversión alimenticia y sus equivalentes en inglés, sin restricciones de fecha.

\subsection{Criterios de inclusión}

Se utilizaron aquellos artículos en los cuales se suplementó Gln exclusivamente a través de la dieta y con animales sanos. Los artículos debían incluir información respecto al número de unidades experimentales por tratamiento (repeticiones), número de animales por unidad experimental y número de animales muestreados por unidad experimental. Los experimentos debían incluir al menos 2 tratamientos (incluyendo el grupo control), nivel de Gln suplementado a través del alimento, porcentaje de lisina y proteína cruda en la dieta. Además, se debía incluir los valores de la media (promedio) y alguna medida de variación, desviación estándar (SD), error estándar (SE) de la variable en estudio.

\subsection{Análisis estadístico}

Para el procesamiento estadístico de los datos se utilizó MIX 2.0 en Microsoft Excel (Bax, 2016). Se determinó el tamaño del efecto global de la suplementación de glutamina sobre la conversión alimenticia calculada con ganancia de peso (CAGP) y conversión alimenticia calculada con peso vivo (CAPV) por diferencia de medias (DM), con intervalos de confianza al 95\%. La heterogeneidad se evaluó por medio del índice de inconsistencia $\left(\mathrm{I}^{2}\right)$ (Higgins \& Thompson, 2002; Lean et al., 2009). Se utilizó un modelo de efectos aleatorios según las recomendaciones de Sauvant et al. (2008) y Borenstein et al. (2011). Se ejecutaron dos meta-análisis a partir de un total de 22 artículos científicos (1814 animales). (Avellaneda et al., 2008; Ayazi, 2014; Bartell \& Batal, 2007; Dai et al., 2009; Dai et al., 2011; Fasina et al., 2010; Hu et al., 2016; Jazideh et al., 2014; Luquetti et al., 2016; Maiorka et al., 2016; Manvailer et al., 2015; Murakami et al., 2007; Muro et al., 2015; Mussini et al., 2012; Namroud et al., 2017; Nascimento et al., 2014; Nassiri Moghaddam \& Alizadeh-Ghamsari, 2013; Olubodun et al., 2015; Ribeiro et al., 2015; Sakamoto et al., 2011; Soltan, 2009; Zavarize et al., 2011). Para explicar la heterogeneidad entre los estudios se realizaron meta-regresiones utilizando como covariables: nivel de inclusión de Gln (\%), edad de inicio de suplementación (días), edad de evaluación (días), duración de la suplementación (días), número de repeticiones por tratamiento, número de pollos muestreados por unidad experimental (repetición), nivel de lisina (\%) y proteína cruda en la dieta (\%).

\section{Resultados}

Los resultados del resumen de la variable analizada conversión alimenticia calculada con base a la ganancia de peso y al peso vivo se presentan en la tabla 1. Se aprecia cómo, por efecto de la suplementación de glutamina, ambos tipos de conversión son más bajos frente al grupo control (sin Gln). El tamaño medio del efecto de la suplementación de Gln sobre la conversión alimenticia expresada en diferencia de medias se muestra en la tabla 2. La suplementación de Gln, en forma altamente significativa, favorece el uso de nutrientes en el pollo de engorde, reduciendo de esta manera la conversión alimenticia.

Tabla 1. Conversión alimenticia tratamiento y control

\begin{tabular}{|c|c|c|c|c|}
\hline \multirow{2}{*}{ Meta-análisis } & \multicolumn{3}{|c|}{ Resumen variable respuesta (g/g) } \\
\cline { 2 - 5 } & \multicolumn{2}{|c|}{ Tratamiento } & \multicolumn{2}{c|}{ Control } \\
\cline { 2 - 5 } & Media & SD & Media & SD \\
\cline { 2 - 5 } & 1,484 & 0,312 & 1,518 & 0,334 \\
\hline CA con ganancia de peso & 1,468 & 0,258 & 1,478 & 0,256 \\
\hline
\end{tabular}

$\mathrm{SD}=$ desviación estándar 
Tabla 2. Tamaño de efecto medio de la suplementación de Gln sobre la CA

\begin{tabular}{|c|c|c|c|c|}
\hline \multirow{2}{*}{ Meta-análisis } & \multicolumn{3}{|c|}{ Tamaño de efecto } \\
\cline { 2 - 5 } & MD & \multicolumn{2}{|c|}{ IC } & P \\
\hline CA con ganancia de peso & $-0,0306$ & $-0,0453$ & $-0,0159$ & 0,00005 \\
\hline CA con peso vivo & $-0,0154$ & $-0,0222$ & $-0,0086$ & 0,00001 \\
\hline
\end{tabular}

$\mathrm{MD}=$ diferencia de medias

$\mathrm{IC}=$ intervalo de confianza

$\mathrm{P}=$ valor de probabilidad

La inconsistencia entre los estudios utilizados fue evaluada a través del test de inconsistencia $\left(\mathrm{I}^{2}\right)$ y se muestra en la tabla 3. Para ambas variables los resultados son altos $(>75 \%)$, mostrando variabilidad entre los estudios utilizados en este trabajo. Debido a los resultados en las pruebas de heterogeneidad se procedió a buscar la explicación de dicha variabilidad a través de meta-regresiones considerando el impacto de algunas co-variables: nivel de inclusión de Gln en la dieta (\%) y edad de inicio de suplementación (días) (tabla 4); edad de evaluación (días) y duración de suplementación (días) (tabla 5); número de repeticiones y número de pollos muestreados por unidad experimental (tabla 6); nivel de lisina (\%) y nivel de proteína cruda (\%) (tabla 7).

Tabla 3. Índice de inconsistencia

\begin{tabular}{|c|c|}
\hline Meta-análisis & $\mathrm{I}^{2}(\%)$ \\
\hline CA con ganancia de peso & 99,99 \\
\hline CA con peso vivo & 81,57 \\
\hline
\end{tabular}

Tabla 4. Meta-regresión para número de repeticiones y número de animales por UE

\begin{tabular}{|c|c|c|c|c|c|c|c|c|}
\hline \multirow{3}{*}{ Meta-análisis } & \multicolumn{4}{|c|}{ Nivel de inclusión de Gln (\%) } & \multicolumn{4}{|c|}{ Edad de inicio de suplementación (d) } \\
\hline & \multicolumn{2}{|c|}{ Intercepto } & \multicolumn{2}{|c|}{ Coef. regresión } & \multicolumn{2}{|c|}{ Intercepto } & \multicolumn{2}{|c|}{ Coef. regresión } \\
\hline & Estimado & $\mathrm{P}$ & Estimado & $\mathrm{P}$ & Estimado & $\mathrm{P}$ & Estimado & $\mathrm{P}$ \\
\hline CA con GP & $-0,0650$ & $<0,001$ & 0,0244 & $<0,001$ & $-0,0407$ & $<0,001$ & $-0,0011$ & 0,039 \\
\hline CA con PV & $-0,0015$ & 0,501 & $-0,0143$ & $<0,001$ & NA & NA & NA & NA \\
\hline
\end{tabular}


Tabla 5. Meta-regresión para edad de evaluación y duración de suplementación

\begin{tabular}{|c|c|c|c|c|c|c|c|c|}
\hline \multirow{2}{*}{ Meta-análisis } & \multicolumn{4}{|c|}{ Edad de evaluación (d) } & \multicolumn{3}{c|}{ Duración de suplementación (d) } \\
\cline { 2 - 9 } & \multicolumn{2}{|c|}{ Intercepto } & \multicolumn{2}{c|}{ Coef. regresión } & \multicolumn{2}{c|}{ Intercepto } & \multicolumn{2}{c|}{ Coef. regresión } \\
\cline { 2 - 9 } & Estimado & $\mathrm{P}$ & Estimado & $\mathrm{P}$ & Estimado & $\mathrm{P}$ & Estimado & $\mathrm{P}$ \\
\hline CA con GP & $-0,0325$ & $<0,001$ & $-0,0004$ & $<0,001$ & $-0,0255$ & $<0,001$ & $-0,0009$ & $<0,001$ \\
\hline CA con PV & $-0,0186$ & $<0,001$ & 0,0002 & 0,1844 & $-0,0300$ & $<0,001$ & 0,0007 & $<0,001$ \\
\hline
\end{tabular}

$\mathrm{CA}=$ conversión alimenticia, $\mathrm{GP}=$ ganancia de peso, $\mathrm{PV}=$ peso vivo, $\mathrm{P}=$ valor de probabilidad.

Tabla 6. Meta-regresión para número de repeticiones y pollos muestreados por UE

\begin{tabular}{|c|c|c|c|c|c|c|c|c|}
\hline \multirow{2}{*}{ Meta-análisis } & \multicolumn{4}{|c|}{ Número de repeticiones } & \multicolumn{3}{c|}{ Número de pollos muestreados por UE } \\
\cline { 2 - 9 } & \multicolumn{2}{|c|}{ Intercepto } & \multicolumn{2}{c|}{ Coef. regresión } & \multicolumn{2}{c|}{ Intercepto } & \multicolumn{2}{c|}{ Coef. regresión } \\
\cline { 2 - 9 } & Estimado & $\mathrm{P}$ & Estimado & $\mathrm{P}$ & Estimado & $\mathrm{P}$ & Estimado & $\mathrm{P}$ \\
\hline CA con GP & 0,0008 & $<0,001$ & $-0,0048$ & $<0,001$ & $-0,1770$ & $<0,001$ & 0,0079 & $<0,001$ \\
\hline CA con PV & $-0,0681$ & $<0,001$ & 0,0095 & $<0,001$ & $-0,0345$ & $<0,001$ & 0,0005 & $<0,001$ \\
\hline
\end{tabular}

$\mathrm{CA}=$ conversión alimenticia, $\mathrm{GP}=$ ganancia de peso, $\mathrm{PV}=$ peso vivo, $\mathrm{P}=$ valor de probabilidad, $\mathrm{UE}=$ unidad experimental.

Tabla 7. Meta-regresión para nivel de lisina y proteína cruda en la dieta

\begin{tabular}{|c|c|c|c|c|c|c|c|c|}
\hline \multirow{3}{*}{ Meta-análisis } & \multicolumn{4}{|c|}{ Nivel de Lisina } & \multicolumn{4}{|c|}{ Nivel de Proteína Cruda } \\
\hline & \multicolumn{2}{|c|}{ Intercepto } & \multicolumn{2}{|c|}{ Coef. Regresión } & \multicolumn{2}{|c|}{ Intercepto } & \multicolumn{2}{|c|}{ Coef. Regresión } \\
\hline & Estimado & $\mathrm{P}$ & Estimado & $\mathrm{P}$ & Estimado & $\mathrm{P}$ & Estimado & $\mathrm{P}$ \\
\hline CA con GP & $-0,2280$ & $<0,001$ & 0,1435 & $<0,001$ & $-0,7700$ & $<0,001$ & 0,0328 & $<0,001$ \\
\hline CA con PV & $-0,0540$ & 0,040 & 0,0327 & 0,123 & 0,0436 & 0,379 & $-0,0026$ & 0,252 \\
\hline
\end{tabular}

$\mathrm{CA}=$ conversión alimenticia, $\mathrm{GP}=$ ganancia de peso, $\mathrm{PV}=$ peso vivo, $\mathrm{P}=$ valor de probabilidad.

\section{Discusión}

La suplementación alimenticia de glutamina en pollos de engorde estimula el desarrollo del tracto gastrointestinal, produce un aumento en el peso relativo del intestino y una mayor longitud en las vellosidades intestinales $(\mathrm{P}<0,05)$ (Abdulkarimi et al., 2019; Bartell \& Batal, 2007; Nassiri Moghaddam y Alizadeh-Ghamsari, 2013). Si la altura de las vellosidades intestinales aumenta de tamaño, especialmente a una edad temprana de su vida, el pollo es capaz de utilizar de manera más eficiente los nu- 
trientes ingeridos y por lo tanto mejorará su desempeño productivo (Namroud et al., 2017). Devi Priya et al. (2010) reportan que la actividad de las enzimas digestivas en pollos broiler, particularmente la amilasa, se incrementa tras la suplementación de Gln. Aves con rápidas tasas de crecimiento presentaron una capacidad alta de secreción de enzimas digestivas y un desarrollo temprano de los órganos gastrointestinales (Nitsan et al., 1991). El aumento en la longitud de las vellosidades intestinales incrementa el desempeño productivo al incrementar la absorción de nutrientes (Xue et al., 2018). En el presente trabajo de meta-análisis se encontró que la suplementación alimenticia de Gln favorece de manera significativa el aprovechamiento de nutrientes en el pollo de engorde reflejado en menores valores de CAGP 1,484 vs. 1,518, y un tamaño medio de efecto de $-0,031$ (DM) $(\mathrm{P}=0,00005)$. En otras palabras, los pollos que no recibieron Gln requieren consumir $31 \mathrm{~g}$ adicionales de alimento para producir $1 \mathrm{~kg}$ de peso ganado en comparación con el grupo que sí recibió Gln. Cuando la variable analizada fue CAPV (1,468 vs. 1,478), se determinó que los pollos sin Gln requieren de $15 \mathrm{~g}$ adicionales de alimento para producir $1 \mathrm{~kg}$ de $\mathrm{PV}(\mathrm{DM}=-0,015$; $\mathrm{P}=0,00001)$. El efecto de la Gln no fue consistente entre los estudios realizados, presentándose valores elevados en el test de inconsistencia $\left(\mathrm{I}^{2}\right), 100 \%$ para CAGP y $81,6 \%$ para CAPV. Los análisis de meta-regresión señalan que todos los factores estudiados tienen efecto significativo sobre la CAGP. Dicha CA aumenta a medida que se incrementa el nivel de $\operatorname{Gln}(\mathrm{P}<0,001)$, número de pollos muestreados por unidad experimental $(\mathrm{P}<0,001)$, nivel de lisina $(\mathrm{P}<0,001)$ y proteína cruda en la $\operatorname{dieta}(\mathrm{P}<0,001)$. La CAGP disminuye a medida que aumenta la

\section{Referencias}

Abdulkarimi, R., Shahir, M. H., \& Daneshyar, M. (2019). Effects of dietary glutamine and arginine supplementation on performance, intestinal morphology and ascites mortality in broiler chickens reared under cold environment. Asian-Australasian Journal of Animal Sciences, 32(1), 110-117. https://doi.org/10.5713/ajas.17.0150

Askanazi, J., Carpentier, Y. A., Michelsen, C. B., Elwyn, D. H., Furst, P., Kantrowitz, L., \& Kin- edad de inicio de la suplementación $(\mathrm{P}=0,039)$, edad de evaluación $(\mathrm{P}<0,001)$, duración de la suplementación $(\mathrm{P}<0,001)$ y número de repeticiones $(\mathrm{P}<0,001)$. Para la CAPV menor cantidad de factores afectan de manera significativa su respuesta, es así que a medida que aumenta la duración de la suplementación $(\mathrm{P}<0,001)$, número de repeticiones $(\mathrm{P}<0,001)$, número de pollos muestreados por unidad experimental $(\mathrm{P}<0,001)$ aumenta la $\mathrm{CA}$. Únicamente se encontró que el aumento del nivel de Gln en la dieta reduce la CAPV $(\mathrm{P}<0,001)$. Respecto a este último punto, estudios individuales reportan que la suplementación de niveles altos de glutamina afecta de manera negativa el desempeño productivo en broilers debido a que causan una depresión del consumo de alimento, como lo muestran los resultados encontrados cuando se utilizó un nivel de $4 \%$ de Gln. Adicionalmente, niveles altos de Gln generan un desbalance con los demás aminoácidos de la dieta afectando su absorción intestinal, a pesar que sí se evidenció un aumento en el tamaño de las vellosidades intestinales (Bartell \& Batal, 2007).

\section{Conclusiones}

La suplementación dietaria de Gln favorece el rendimiento productivo en pollos de engorde al reducir la conversión alimenticia independientemente de la forma de medición (calculado con ganancia de peso o con peso vivo). Sin embargo, se debe considerar el efecto de ciertos factores que de manera significativa tienen impacto sobre esta variable respuesta, ya sea para el diseño de un experimento o para la fabricación de alimentos balanceados que incluyan Gln en su formulación.

ney, J. M. (1980). Muscle and plasma amino acids following injury: influence of intercurrent infection. Annals of Surgery, 192(1), 78.

Avellaneda, Y., Hernández, J., Ariza, C., \& Afanador, T. (2008). Efecto de la suplementación de L-glutamina y L-glutamato (Aminogut ${ }^{\circledR}$ ) sobre el crecimiento temprano de pollos de engorde. Revista de la Facultad de Medicina Veterinaria y de Zootecnia, 55(2), 77-90. 
Ayazi, M. (2014). The effect of dietary glutamine supplementation on performance and blood parameter, carcass characteristics, quality and characteristics meat of broiler chickens under continuous heat stress condition. International Journal of Farming and Allied Sciences, 3(12), 1234-1242.

Bartell, S. M., \& Batal, A. B. (2007). The effect of supplemental glutamine on growth performance, development of the gastrointestinal tract, and humoral immune response of broilers. Poultry Science, 86(9), 1940-1947. https://doi. org/10.1093/ps/86.9.1940

Bax, L., 2016. MIX 2.0 - Professional software for meta-analysis in Excel. Version 2.0.1.5. BiostatXL. Obtenido de: https://www.meta-analysis-made-easy.com/

Borenstein, M., Hedges, L. V., Higgins, J. P., \& Rothstein, H. R. (2011). Introduction to meta-analysis. John Wiley \& Sons.

Calder, P. C., \& Yaqoob, P. (1999). Glutamine and the immune system. Amino acids, 17(3), 227241. https://doi.org/10.1007/BF01366922

Dai, S. F., Gao, F., Zhang, W. H., Song, S. X., Xu, X. L., \& Zhou, G. H. (2011). Effects of dietary glutamine and gamma-aminobutyric acid on performance, carcass characteristics and serum parameters in broilers under circular heat stress. Animal Feed Science and Technology, 168(1-2), 51-60. https:// doi.org/10.1016/j.anifeedsci.2011.03.005

Dai, S. F., Wang, L. K., Wen, A. Y., Wang, L. X., \& Jin, G. M. (2009). Dietary glutamine supplementation improves growth performance, meat quality and colour stability of broilers under heat stress. British Poultry Science, 50(3), 333-340. https://doi. org/10.1080/00071660902806947

Devi Priya, K., Selvaraj, P., Nanjappan, K., \& Jayachandran, S. (2010). Oral supplementation of putrescine and 1-glutamine on the growth performance, immunity, intestinal enzymes in the broiler chickens. Tamilnadu Journal of Veterinary and Animal Sciences, 6(5), 250-254.
Fasina, Y. O., Bowers, J. B., Hess, J. B., \& McKee, S. R. (2010). Effect of dietary glutamine supplementation on Salmonella colonization in the ceca of young broiler chicks. Poultry Science, 89(5), 1042-1048. https://doi.org/10.3382/ ps.2009-00415

Higgins, J. P., \& Thompson, S. G. (2002). Quantifying heterogeneity in a meta-analysis. Statistics in Medicine, 21(11), 1539-1558. https://doi. org/10.1002/sim.1186

Hu, H., Bai, X., Shah, A. A., Wen, A. Y., Hua, J. L., Che, C. Y., \& Dai, S. F. (2016). Dietary supplementation with glutamine and $\gamma$-aminobutyric acid improves growth performance and serum parameters in 22-to 35-day-old broilers exposed to hot environment. Journal of Animal Physiology and Animal Nutrition, 100(2), 361-370. https://doi.org/10.1111/jpn.12346

Jazideh, F., Farhoomand, P., Daneshyar, M., \& Najafi, G. (2014). The effects of dietary glutamine supplementation on growth performance and intestinal morphology of broiler chickens reared under hot conditions. Turkish Journal of Veterinary and Animal Sciences, 38(3), 264270. https://doi.org/10.3906/vet-1210-32

Labow, B. I., Souba, W. W., \& Abcouwer, S. F. (2001). Mechanisms governing the expression of the enzymes of glutamine metabolism-glutaminase and glutamine synthetase. The Journal of Nutrition, 131(9), 2467S-2474S. https:// doi.org/10.1093/jn/131.9.2467S

Lean, I. J., Rabiee, A. R., Duffield, T. F., \& Dohoo, I. R. (2009). Invited review: Use of meta-analysis in animal health and reproduction: Methods and applications. Journal of Dairy Science, 92(8), 3545-3565. https://doi.org/10.3168/ jds.2009-2140

Luquetti, B. C., Alarcon, M. F., Lunedo, R., Campos, D. M., Furlan, R. L., \& Macari, M. (2016). Effects of glutamine on performance and intestinal mucosa morphometry of broiler chickens vaccinated against coccidiosis. Scientia Agricola, 73(4), 322-327. http://dx.doi. org/10.1590/0103-9016-2015-0114 
Maiorka,A., Silva,A. V., Santin, E., Dahlke, F., Bruno, L. D., Boleli, I. C., \& Trautenmuller, H. (2016). Effect of broiler breeder age and glutamine supplementation on the development of the intestinal mucosa of 7-day-old chicks. Revista Brasileira de Ciência Avícola, 18(1), 17-22. http:// dx.doi.org/10.1590/1516-635X1801017-022

Manvailer, G. V., Kiefer, C., de Souza, K. M., Marçal, D. A., Paiva, L. L., Rodrigues, G. P., \& Ozelame, A. M. (2015). Glutamine for broilers reared in hot environment. Archivos de Zootecnia, 64(248), 377-382.

Menconi, A., Kallapura, G., Hernandez-Velasco, X., Latorre, J., Morgan, M., Pumford, N. R., \& Barton, J. (2013). Effect of glutamine supplementation associated with probiotics on salmonella typhimurium and nitric oxide or glutamine with perinatal supplement on growth performance and intestinal morphology in broiler chickens. Clinical Microbiology, 2(120), 1-7. http://dx. doi.org/10.4172/2327-5073.1000120

Murakami, A. E., Sakamoto, M. I., Natali, M. R., Souza, L. M., \& Franco, J. R. (2007). Supplementation of glutamine and vitamin $\mathrm{E}$ on the morphometry of the intestinal mucosa in broiler chickens. Poultry Science, 86(3), 488-495. https://doi.org/10.1093/ps/86.3.488

Muro, E. M., Pelícia, V. C., Vercese, F., de Souza, I. G., Pimenta, G. M., Oliveira, R. D., \& Sartori, J. R. (2015). Aditivos fitogênicos e glutamina mais ácido glutâmico na dieta de frangos desafiados com coccidiose. Agrarian, 8(29), 304-311.

Mussini, F. J., Goodgame, S. D., Lu, C., Bradley, C. D., Fiscus, S. M., \& Waldroup, P. W. (2012). A nutritional approach to the use of anticoccidial vaccines in broilers: glutamine utilization in critical stages of immunity acquisition. International Journal of Poultry Science, 11(4), 243-246.

Namroud, N. F., Shivazad, M., Zaghari, M., Madadgar, O., \& Nourijelyani, K. (2017). Impact of dietary glutamine on amino acid digestibility values and intestinal morphometric parameters in neonate chicks. South African Journal of
Animal Science, 47(4), 440-453. http://dx.doi. org/10.4314/sajas.v47i4.3

Nascimento, G. M., Leandro, N. S., Café, M. B., Stringhini, J. H., Andrade, M. A., Martinez, K. D., \& Mascarenhas, A. G. (2014). Performance and intestinal characteristics of broiler chicken fed diet with glutamine in the diet without anticoccidials agents. Revista Brasileira de Saúde e Produção Animal, 15(3), 637-648. http://dx. doi.org/10.1590/S1519-99402014000300011

Nassiri Moghaddam, H., \& Alizadeh-Ghamsari, A. H. (2013). Improved performance and small intestinal development of broiler chickens by dietary L-glutamine supplementation. Journal of Applied Animal Research, 41(1), 1-7. https:// doi.org/10.1080/09712119.2012.738214

Newsholme, P. (2001). Why is L-glutamine metabolism important to cells of the immune system in health, postinjury, surgery or infection? The Journal of Nutrition, 131(9), 2515S-2522S. https://doi.org/10.1093/jn/131.9.2515S

Nitsan, Z., Dunnington, E. A. \& Siegel, P. B., 1991. Organ growth and digestive enzyme levels to fifteen days of age in lines of chickens differing in body weight. Poultry Science, 70(10), 20402048. https://doi.org/10.3382/ps.0702040

Olubodun, J. O., Zulkifli, I., Farjam, A. S., Hair-Bejo, M., \& Kasim, A. (2015). Glutamine and glutamic acid supplementation enhances performance of broiler chickens under the hot and humid tropical condition. Italian Journal of Animal Science, 14(1), 3263. https://doi.org/10.4081/ ijas.2015.3263

Phanvijhitsiri, K., Musch, M. W., Ropeleski, M. J., \& Chang, E. B. (2006). Heat induction of heat shock protein 25 requires cellular glutamine in intestinal epithelial cells. American Journal of Physiology-Cell Physiology, 291(2), C290-C299.https://doi.org/10.1152/ajpce11.00225 .2005

Pohlenz, C., Buentello, A., Bakke, A. M., \& Gatlin III, D. M. (2012). Free dietary glutamine improves intestinal morphology and increases en- 
terocyte migration rates, but has limited effects on plasma amino acid profile and growth performance of channel catfish Ictalurus punctatus. Aquaculture, 370, 32-39. https://doi.org/10.1016/j.aquaculture.2012.10.002

Ribeiro, J. V., Albino, L. F., Rostagno, H. S., Hannas, M. I., Ribeiro, C. L., Vieira, R. A., \& da Silva, D. L. (2015). Effects of dietary L-glutamine or L-glutamine plus L-glutamic acid supplementation programs on the performance and breast meat yield uniformity of 42-d-Old Broilers. Brazilian Journal of Poultry Science, 17(SPE), 93-98. http://dx.doi.org/10.1590/1516-635XSPECIALISSUENutrition-PoultryFeedingAdditives093-098

Roth, E. (2008). Nonnutritive effects of glutamine. The Journal of Nutrition, 138(10), 2025 S-2031S.https://doi.org/10.1093/ jn/138.10.2025S

Sakamoto, M. I., Faria, D. E., Nakagi, V. S., Negrão, J. A., Araújo, R. B., Souza, K. M., \& Previero, T. C. (2011). Utilization of glutamine, associated with glutamic acid, on development and enzymatic activity in broiler chickens. Arquivo Brasileiro de Medicina Veterinária e Zootecnia, 63(4), 962-972. http://dx.doi.org/10.1590/ S0102-09352011000400023

Salmanzadeh, M., Ebrahimnezhad, Y., Aghdam Shahryar, H., \& Ghiasi Ghaleh-Kandi, J. (2016). The effects of in ovo feeding of glutamine in broiler breeder eggs on hatchability, development of the gastrointestinal tract, growth performance and carcass characteristics of broiler chickens. Archives Animal Breeding, 59(2), 235-242. https://doi.org/10.5194/ aab-59-235-2016

Sauvant, D., Schmidely, P., Daudin, J. J., \& St-Pierre, N. R. (2008). Meta-analyses of experimental data in animal nutrition. Animal, 2(8), 1203-1214. https://doi.org/10.1017/ S1751731108002280

Shakeri, M., Oskoueian, E., Najafi, P., \& Ebrahimi, M. (2015). Impact of glutamine in drinking water on performance and intestinal morphology of broiler chickens under high stocking density. Ístanbul Üniversitesi Veteriner Fakültesi Dergisi, 42(1), 51-56. https://doi.org/10.16988/iuvfd.2016.42317

Sifa, D., Bai, X., Zhang, D., Hu, H., Wu, X., Wen, A., Zhao, L. (2018). Dietary glutamine improves meat quality, skeletal muscle antioxidant capacity and glutamine metabolism in broilers under acute heat stress. Journal of Applied Animal Research, 46(1), 1412-1417. https://doi.org/10.108 0/09712119.2018.1520113

Soares, A. D., Costa, K. A., Wanner, S. P., Santos, R. G., Fernandes, S. O., Martins, F. S., \& Cardoso, V. N. (2014). Dietary glutamine prevents the loss of intestinal barrier function and attenuates the increase in core body temperature induced by acute heat exposure. British Journal of Nutrition, 112(10), 1601-1610. https:// doi.org/10.1017/S0007114514002608

Soltan, M. A. (2009). Influence of dietary glutamine supplementation on growth performance, sma11 intestinal morphology, immune response and some blood parameters of broiler chickens. International Journal of Poultry Science, 8(1), 60-68.

Wang, H., Zhang, C., Wu, G., Sun, Y., Wang, B., He, B., .\& Wu, Z. (2014). Glutamine enhances tight junction protein expression and modulates corticotropin-releasing factor signaling in the jejunum of weanling piglets. The Journal of $\mathrm{Nu}$ trition, 145(1), 25-31. https://doi.org/10.3945/ jn. 114.202515

Wischmeyer, P. E. (2002). Glutamine and heat shock protein expression. Nutrition, 18(3), 225-228. https://doi.org/10.1016/S0899-9007(01)00796-1

Wu, G., Bazer, F. W., Johnson, G. A., Knabe, D. A., Burghardt, R. C., Spencer, T. E., \& Wang, J. J. (2011). Triennial Growth Symposium: important roles for L-glutamine in swine nutrition and production. Journal of Animal Science, 89(7), 2017-2030. https://doi.org/10.2527/ jas.2010-3614

Xue, G. D., Barekatain, R., Wu, S. B., Choct, M., \& Swick, R. A. (2018). Dietary L-glutamine su- 
pplementation improves growth performance, gut morphology, and serum biochemical indices of broiler chickens during necrotic enteritis challenge. Poultry Science, 97(4), 1334-1341. https:// doi.org/10.3382/ps/pex444

Yi, G. F., Allee, G. L., Knight, C. D., \& Dibner, J. J. (2005). Impact of glutamine and oasis hatchling supplement on growth performance, small intestinal morphology, and immune response of broilers vaccinated and challenged with Eimeria maxima. Poultry Science, 84(2), 283-293. https://doi.org/10.1093/ps/84.2.283
Zavarize, K. C., Sartori, J., Pelícia, V., Pezzato, A., Araujo, P., Stradiotti, A., \& Madeira, L. (2011). Glutamina e nucleotídeos na dieta de frangos de corte criados no sistema alternativo. Archivos de Zootecnia, 60(232), 913-920.

Zhong, X., Li, W., Huang, X., Wang, Y., Zhang, L., Zhou, Y., ...\& Wang, T. (2012). Effects of glutamine supplementation on the immune status in weaning piglets with intrauterine growth retardation. Archives of Animal Nutrition, 66(5), 347-356. https://doi. org/10.1080/1745039X.2012.683325 